\title{
Relación entre la satisfacción laboral y el trato digno que brinda el profesional de Enfermería al paciente que acude a una institución privada de salud de IV nivel de atención, Lima 2013
}

\author{
Relationship between job satisfaction and decent treatment gives the nurse the patient to go to a \\ private health facility level IV care Lima - 2013
}

\author{
Danitza Raquel Casildo Bedón ${ }^{1}$
}

\begin{abstract}
RESUMEN
Objetivo: Establecer la relación entre la satisfacción laboral y el trato digno que brinda el profesional de Enfermería al paciente que acude a una institución privada de salud de IV nivel de atención en la ciudad de Lima. Metodología: El estudio fue de diseño no experimental; tipo cuantitativo, descriptivo, correlacional y transversal. La población estuvo constituida por 57 profesionales de Enfermería que trabajan en el área asistencial de la Clínica San Felipe, durante el último año. En la recolección de datos se utilizó dos instrumentos de medición, para la variable satisfacción laboral se adaptó y modificó el cuestionario de Robbins desarrollado en el año 2000; para la variable trato digno se utilizó una guía de observación elaborada por la investigadora teniendo como referencia la encuesta indicador de calidad presentada por la Cruzada Nacional de calidad de los servicios de salud. Para establecer la relación entre las variables se utilizó el estadístico de correlación de Rho Spearman $\left(\mathrm{r}_{\mathrm{s}}\right)$. Resultados: Se evidenció que la satisfacción laboral no tuvo relación significativa con el trato digno; según la prueba Rho Spearman 0,042 y el valor $p=0,758$. Sin embargo se encontró una correlación positiva significativa para la dimensión: naturaleza de contenido de trabajo y la variable trato digno que brinda el profesional de enfermería. Conclusión: No existe correlación significativa entre la satisfacción laboral y el trato digno que brinda el profesional de Enfermería.
\end{abstract}

Palabras clave: Trato digno, satisfacción laboral, enfermería, cuidado humano.

\begin{abstract}
Objective: To establish the relationship between job satisfaction and decent treatment provides professional nursing patient who attends a private institution level IV health care in the city of Lima. Methodology: The study was non-experimental design; quantitative, descriptive, correlational and cross-sectional. The study population consisted of 57 nursing professionals working in the health care area of San Felipe Clinic during the past year. In data collection Two measuring instruments used for job satisfaction variable is adapted and modified Robbins questionnaire developed in 2000; Variable dignified treatment for an observation guide developed by the researcher with reference to the quality indicator survey by the National Crusade quality health services was used. To establish the relationship between the variables statistical Spearman Rho correlation (rs) was used. Results: We showed that job satisfaction had no significant relationship with dignity; according to Spearman Rho test 0.042 and $p=0.758$ value. Nature of work content and dignified treatment variable that provides professional nursing: Though a significant positive correlation was found for the dimension. Conclusion: There is no significant correlation between job satisfaction and decent treatment provides professional nursing.
\end{abstract}

Keywords: Dignified treatment, job satisfaction, nursing, human care.

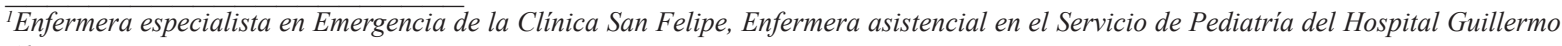
Almenara Irigoyen

Correspondencia: danitza1535@hotmail.com 
Relación entre la satisfacción laboral y el trato digno que brinda el profesional de Enfermería al paciente que acude a una institución privada de salud de IV nivel de atención, Lima 2013

\section{INTRODUCCIÓN}

El interés de las empresas de salud por mantener una mejora continua de la calidad en la prestación de los servicios, se ha incrementado en los últimos años. Existe la necesidad de buscar una participación activa, donde se integre a los trabajadores en equipo, para lograr el cumplimiento de los objetivos de la organización.

No podría ser de otra forma, ya que a fin de cuentas las organizaciones son "medios" para lograr "fines", en los que la necesaria dinámica, creativa y activa participación del hombre, incide en su eficacia, eficiencia y competitividad de una manera trascendental y permiten que hoy sea reconocido no solo como "recurso" sino como potencial y eje central de la ventaja competitiva de organizaciones y naciones.

El hombre vive en sociedad y de ella emanan beneficios que brindan o no satisfacción que influyen en su interior, generando actitudes y comportamientos consecuentes con ellas.

La satisfacción laboral es la actitud que asume la persona ante su trabajo, esta se reflejará en actitudes, sentimientos, estados de ánimo y comportamientos en relación a su actividad laboral. Si la persona está satisfecha con su trabajo, responderá adecuadamente a las exigencias de éste; si, por el contrario, está insatisfecha no será capaz de realizar su labor con eficiencia y calidad. Además, la insatisfacción laboral se refleja en todas la esferas de la vida del trabajador.

El presente trabajo de investigación tiene el objetivo de establecer la relación entre la satisfacción laboral y el trato digno que brinda el profesional de Enfermería al paciente que acude a una institución privada de salud de IV nivel de atención en Lima, en el año 2013.

\section{MATERIAL Y MÉTODOS}

El estudio es de diseño no experimental; tipo cuantitativo, descriptivo, correlacional y transversal. Se realizó en la Clínica San Felipe S. A. ubicada en Lima, en los distritos de Jesús María y La Molina. Jr. Costa Rica 235/ Jesús María, la Clínica San Felipe y el Centro Médico en la Av. Javier Prado Este 4841 La Molina, Lima.

La población estuvo constituida por 143 profesionales de Enfermería y la selección de la muestra se realizó a través de muestreo no probabilístico a criterio del investigador, se basó en los siguientes criterios de inclusión fueron: profesionales de Enfermería con un mínimo de un año de experiencia, trabajando en la clínica y que labora en el área asistencial.

Asimismo, los criterios de exclusión incluyeron a los profesionales de Enfermería que no aceptaron participar en el estudio o que se encuentran con licencias o permisos. La muestra estuvo conformada por 57 enfermeras asistenciales.

Se utilizó dos instrumentos de medición. Para la variable satisfacción laboral, la investigadora modificó el cuestionario de Robbins desarrollado el año 2000; adoptándola a la muestra de estudio que estuvo constituida por 30 preguntas. La escala de opciones tiene una puntuación de 1 a 5 , sumando un puntaje total de 150 .

Se identifican tres escalas: no satisfecho, medianamente satisfecho y satisfecho, identificado con un puntaje de 30 a 70 , de 71 a 110 y de 111 a 150 respectivamente.

Asimismo, para medir la variable trato digno, se utilizó una guía de observación, la cual también fue modificada para la población de estudio, esta fue redactada y guiada por las encuestas de los estudios de investigación referidos en los antecedentes y la encuesta indicador de calidad presentada por la Cruzada Nacional por la calidad de los servicios de salud. Esta guía consta de 15 ítems, cada uno con 5 opciones de selección; sumando un puntaje total de 75 .

Las escalas de interpretación son cinco: nunca brinda trato digno, casi nunca brinda trato digno, a veces brinda trato digno, casi siempre brinda trato digno y siempre brinda trato digno con los puntajes de 0 a 15 , de 16 a 30 , de 31 a 45 , de 46 a 60 y de 61 a 75 respectivamente.

Donde "siempre" significa que todas las veces realiza la actividad; "Casi siempre" significa que de todas las veces, en una oportunidad lo realizó parcialmente; "A veces" significa que no lo realizó todas las veces; "Casi nunca" significa que solo una vez lo hizo y "Nunca" significa que no realizó la actividad $\mathrm{o}$ ítem en ninguna de las observaciones realizadas.

Ambas encuestas fueron sometidas a juicio de tres expertos para su validación. Luego se aplicó una prueba piloto en una muestra de similares características al de la población seleccionada, obteniendo el valor de 0.86 en el coeficiente alfa de Cronbach para la encuesta de satisfacción laboral. 
Para la encuesta de trato digno, se obtuvo un puntaje de 0.9 en el alfa de Cronbach, estas puntuaciones indican la alta confiabilidad de ambos instrumentos (Jara, 2010).
Los datos obtenidos fueron procesados al programa SPSS versión 15, con el coeficiente de correlación por rangos ordenados de Spearman para su procesamiento.

\section{RESULTADOS}

Tabla 1

Características de la muestra en estudio de una institución privada de salud nivel IV. Lima-2013.

\begin{tabular}{cccccc}
\hline Años de edad: & $N^{\circ}$ & $\%$ & Área en el que labora: & $N^{\circ}$ & $\%$ \\
\hline $24-28$ años & 3 & 5.3 & Sala de operaciones & 5 & 8,8 \\
$29-33$ años & 10 & 17.6 & Recuperación & 3 & 5,3 \\
$34-37$ años & 8 & 14.1 & Emergencia & 7 & 12,3 \\
$38-41$ años & 9 & 15.9 & Hospitalización 2 & 9 & 15,8 \\
$42-45$ años & 12 & 21.1 & Hospitalización 3 & 7 & 12,3 \\
$46-49$ años & 8 & 14.2 & Hospitalización 4 & 6 & 10,5 \\
$50-53$ años & 3 & 5.3 & UCIN & 7 & 12,3 \\
$54-57$ años & 3 & 5.4 & UCl & 8 & 14,0 \\
$58-62$ años & 0 & 0 & Neonatología & 3 & 5,3 \\
$63-67$ años & 1 & 1.8 & Emergencia -Camacho & 2 & 3,5 \\
Total & 57 & 100,0 & Total & 57 & 100,0 \\
\hline
\end{tabular}

Se puede apreciar, en la tabla 1 , que del $100 \%$ de enfermeras de la muestra de estudio, el mayor porcentaje se encuentra entre los 42 y 45 años, con un $21.1 \%$ y predomina el grupo que trabaja en hospitalización 2 con un $15,8 \%$, seguido del grupo que labora en UCI con un $14 \%$.

Tabla 2

Correlación entre satisfacción laboral y el trato digno que brinda el profesional de Enfermería al paciente que acude a una institución privada de salud de IV nivel de atención, Lima 2013.

\begin{tabular}{llcc}
\hline & & Satisfacción laboral & Trato digno \\
\hline \multirow{2}{*}{ Satisfacción } & Coeficiente de correlación de Rho Spearman & 1 &, 042 \\
Laboral & Sig. (bilateral) & &, 758 \\
& $\mathrm{~N}$ & 57 & 57 \\
Trato & Coeficiente de correlación de Rho Spearman &, 042 & 1 \\
Digno & Sig. (bilateral) &, 758 & \\
& \multicolumn{1}{c}{$N$} & 57 & 57 \\
\hline
\end{tabular}

La tabla 2 señala que según la prueba de Rho Spearman, el valor $\mathrm{p}=0,758>\alpha$, revelando que la relación entre las variables de estudio no fue significativa; y el grado de correlación de 0,042 fue muy baja o débil. Ante las evidencias encontradas se acepta la hipótesis nula y se rechaza la hipótesis alterna, concluyendo que la satisfacción laboral no tiene relación positiva con el trato digno que brinda el profesional de Enfermería. 
Relación entre la satisfacción laboral y el trato digno que brinda el profesional de Enfermería al paciente que acude a una institución privada de salud de IV nivel de atención, Lima 2013

\section{DISCUSIÓN}

La evidencia de un alto nivel de insatisfacción al $47.4 \%$ es revelador de la forma cómo las enfermeras experimentan las características de su trabajo, y el significado que atribuyen al producto de sus esfuerzos individuales y compartidos.

Para Chiavenato (1996), los indicadores de insatisfacción encontrados, entre las enfermeras, están asociados a la percepción de existir una falta de correspondencia entre el nivel de preparación, calidad de trabajo, cantidad de trabajo, estímulos materiales y remuneración salarial. Uno de los indicadores más comunes asociados a la insatisfacción laboral de los profesionales de Enfermería, en esta dimensión, es el bajo salario; comparado con el nivel de preparación, la cantidad y calidad del trabajo. Al respecto, existe similitud con los resultados obtenidos por Herrera y Manrique (2008), en el estudio condiciones laborales y grado de satisfacción de profesionales de Enfermería; los autores encontraron que, en los nuevos sistemas, un alto número de enfermeras/os perciben que sus remuneraciones son menores en relación al valor de su trabajo, identifican estar mal remuneradas/os; situación que no les permite una vida socialmente decorosa; los ingresos que perciben no están en relación a sus necesidades básicas y son, por tanto, insuficientes.

Las enfermeras del estudio experimentan que la alta capacitación que ellas mismas se autogestionan y la complejidad del trabajo que desarrollan, no gozan de un justo reconocimiento.

En tal sentido, Iriarte (2012) y Alba, Salcedo, Zárate e Higuera (2008), en sus estudios respecto al salario, encontraron cifras de insatisfacción similares, con un $35,8 \%$ de profesionales insatisfechos.

La insatisfacción existente, en el grupo de estudio, revela, entre otros aspectos, que todavía las autoridades así como los administradores de las instituciones de salud, aún no han valorado en la correcta dimensión el trabajo que realiza el profesional de Enfermería. Esta situación es similar en los países en vías de desarrollo (Cifuentes, 2012), y en instituciones de salud públicas y privadas, tal como lo reportan Fernández y Paravic (2003), en el estudio sobre satisfacción laboral en enfermeras de hospitales públicos y privados de Chile, quienes encontraron que los factores remuneraciones, promociones y/o ascensos son aquellos con los que se encuentran más insatisfechas las enfermeras de ambos grupos.
Por otro lado, las bajas remuneraciones salariales, obligan, en cierta medida, a que las enfermeras decidan trabajar en dos o más instituciones de salud, arriesgando su salud debido a la falta de descanso, se produce disminución de la calidad y cantidad de trabajo y se posterga la vida familiar.

La condición de bienestar es la segunda dimensión de baja satisfacción en el personal de Enfermería, porque solo el $10 \%$ de las enfermeras refirió estar satisfecha, comparada a un $22 \%$ quienes refieren insatisfacción.

En tal sentido, Ruzafa, Madrigal, Velandrino y López (2008) encontraron, en su estudio, que las dimensiones asociadas a una satisfacción laboral más alta fueron las relaciones con sus compañeros de trabajo y las relaciones con los jefes. Aunque en el presente estudio, las relaciones con los jefes tienen puntuaciones bajas de satisfacción, porque ante la pregunta si los supervisores brindan al grupo la información de la calidad con que realizan su tarea, un significativo porcentaje $(40 \%)$ refirió entre nunca y casi nunca.

Más de la mitad de las enfermeras dijeron que nunca y casi nunca están satisfechas con las condiciones de desarrollo personal y profesional, la misma respuesta se obtuvo en relación al fomento de condiciones de bienestar por parte del centro laboral: alimentación, transporte, facilidades para la atención de la salud.

En cuanto al indicador de trabajo en equipo, se observa que más de la mitad de la población en estudio se siente satisfecha; resultado concordante con la afirmación de Cifuentes (2012), quien expresó que los niveles altos de trabajo en equipo y una dotación de personal adecuada, dentro de los hospitales, conduce a una mayor satisfacción.

Kalisch, Lee y Rochman (2012) encontraron, dentro del personal de Enfermería, mayores puntuaciones en el trabajo interdisciplinario en equipo, mayor propensión a estar satisfechos con sus puestos de trabajo y obtenían al mismo tiempo menores puntuaciones de Burnout.

El constructo trato digno cuenta con limitada información; sin embargo, los elementos que componen esta variable tienen su equivalente semántico en las expresiones "cuidado humanizado", "cuidado humano", "respeto a la dignidad humana", "ética del cuidado" que corresponden a los atributos de la calidad del cuidado (Ortega, 2004). 
El trato digno es equivalente a un comportamiento altruista, solidario, con delicado respeto por el otro; es desarrollar empatía, interés genuino por las personas. Estas características pueden materializarse en la práctica del cuidado si las enfermeras poseen habilidades cognitivas, técnicas y personales. El paciente espera respeto, tolerancia, información sencilla, paciencia, calor en la mirada, proximidad. Recibir trato frío, indiferencia o desinterés es la ausencia significativa de la profesionalidad que conlleva cuidar con una visión holística. (Mahomed, John y Patterson (2012), Hernández, Ochando, Mora, Martínez y López (2005)).

Valorar las expectativas del paciente en su totalidad, está por encima de las habilidades científico-técnicas (Dios, Alba, Ruiz, Jiménez y Rodríguez, 2013).

Eriksen, citado por Ortega (2004), aborda cinco dimensiones de la calidad del cuidado: arte del cuidado, la calidad técnica del cuidado, la disponibilidad, la continuidad del cuidado y el resultado del cuidado. El arte del cuidado de Enfermería se enfoca hacia la cantidad de cuidado demostrado por el proveedor del cuidado de salud, incluyendo los elementos de la cortesía en el trato, la comprensión del paciente, la paciencia con el usuario, la atención al paciente, la amabilidad, el ser cordial con el paciente, el uso de terminología clara, así como la disponibilidad de escuchar los temores del paciente y el cuidado de la privacidad proporcionada por la enfermera al paciente.

Parra, Guáqueta y Triana (2012), en su modelo de cuidado de Enfermería H.A.N.C., definen cuatro dimensiones del cuidado para el paciente crítico: dimensión ética, científico-clínica, relación interpersonal y de percepción. En cada dimensión establecen las correspondientes categorías de cuidado, a partir de las cuales se definen los indicadores de calidad del cuidado.

Al hablar de indicadores de la calidad del cuidado en UCI: desde la dimensión ética, resaltan el respeto a la vida, el respeto a la dignidad, a la autonomía, a la privacidad y la aplicación de los principios éticos de beneficencia, no maleficencia, autonomía y justicia.

En los indicadores de la calidad del cuidado en UCI, desde la dimensión relación interpersonal, se identifican los indicadores de comprensión empática, uso de códigos que permiten la interacción, la capacidad de escuchar, comunicación con la familia y respeto a la dignidad. Finalmente, dentro de los indicadores de calidad del cuidado en UCI: desde la dimensión de percepción, las autoras usan el instrumento.
Gómez (2008), realiza un análisis para aclarar conceptos básicos; concluyendo que el cuidado de Enfermería está relacionado directamente con los factores o determinantes del cuidado; entre ellos señaló a la beneficencia, la no maleficencia, la autonomía y justicia, el respeto a la intimidad, la privacidad, la capacidad para escuchar, la confidencialidad, la veracidad y el cuidado continuo, valores que deben estar impregnados en el cuidado de la enfermera.

En el presente estudio, se puede observar que la mayoría de los profesionales de Enfermería "siempre" brinda trato digno, y solo el 19.3\% estuvo situado en la escala, "casi siempre" brinda trato digno. Resultados similares mostraron García y Cortés (2012), en la investigación sobre la percepción del usuario del trato digno que brinda la enfermera en un hospital del estado de México, donde el 91\% de los encuestados señaló recibir trato adecuado por parte de las enfermeras.

Los indicadores de la variable trato digno que obtuvieron elevados porcentajes de fueron: saludo al paciente cuando ingresa a la habitación, pide el consentimiento del paciente en cada una de las actividades que va a realizar, brinda condiciones de seguridad ante infecciones o lesiones potenciales a todos por igual, se empeña en realizar cada acto de cuidado evitando todo daño al paciente y no descubre innecesariamente el cuerpo del paciente $(86 \%, 77.2 \%$, $75.4 \%, 73.7 \%$ y $70.2 \%$ respectivamente).

En ese contexto, Delgado, Álvarez y Aliaga (2007), al estudiar la calidad de atención de Enfermería desde la percepción del usuario, encontraron que más de la mitad de los encuestados mostró porcentajes de aprobación en las categorías "siempre" y "casi siempre", las características que resaltaron fueron: el saludo cordial y respetuoso al ser atendidos, la capacidad de escuchar, las respuestas a todas las preguntas respecto a su enfermedad, respeto a la privacidad y actuar mostrando seguridad durante su atención.

Molina (2011), en la investigación "Evaluación de la calidad del cuidado de Enfermería en un servicio de hospitalización domiciliaria en Bogotá, Colombia, encontró un alto nivel de satisfacción en la población estudiada, respecto a los siguientes indicadores: "Es honesta con el paciente en cuanto a su condición médica", (97\%). "La enfermera le da información clara y precisa sobre su situación de salud" (80\%), "La enfermera lo escucha con atención" (98\%), "Infunde ánimo al paciente identificando elementos positivos relacionados con el estado y el tratamiento del paciente" (86\%). "Logra tratar al paciente como 
una persona" (99\%). "Se presenta al paciente, le explica el procedimiento a realizar" (93\%). "Tiene una proximidad estable con el paciente" (83\%). "La enfermera realiza los procedimientos con seguridad" (98\%). "Proporciona buen cuidado físico al paciente" (91\%). (Ortega, 2004), los resultados relacionados con la evaluación del cuidado de Enfermería, respecto a la dimensión de satisfacción, mostraron que las puntuaciones más altas se relacionaron con la amabilidad, trato recibido como persona y la intimidad proporcionada.

Al correlacionar las dimensiones de la satisfacción laboral y el trato digno que brinda el profesional de enfermería al paciente crítico se encontró una correlación positiva significativa entre la dimensión naturaleza de contenido de trabajo y trato digno con $\mathrm{P}=0,012$, para las demás dimensiones de la satisfacción laboral no se encontró correlación significativa.

Esto puede ser explicado por el sentido de compromiso, responsabilidad y ética del profesional de Enfermería, para con las personas a quienes brinda cuidados, y que está por encima de la falta de reconocimiento por parte de jefes, de las bajas remuneraciones salariales en relación al tipo, cantidad y trabajo que realiza, a la falta de condiciones adecuadas de desarrollo personal y profesional e incentivos. Prueba de ello es que aun cuando en la dimensión condiciones de trabajo las enfermeras reportaron estar satisfechas, no se encontró asociación significativa con el trato digno. 


\section{REFERENCIAS BIBLIOGRÁFICAS}

Alba, A., Salcedo, R., Zárate, R., Higuera, F. (2008). Satisfacción laboral del personal de enfermería en el Hospital General de México. Enferm. Inst. Mex. 16 (3) 155-160 Recuperado de: http://www. imss.gob.mx/publicaciones/salud/enfermeria/ Documents/305Satisfaccionlaboral.pdf

Cifuentes, J. (2012). Satisfacción laboral en enfermería en una institución de salud de cuarto nivel de atención. (Tesis Maestría). Universidad Nacional de Colombia. Publicado en: http://www.bdigital.unal.edu. co/8907/1/539680.2012.pdf

Chiavenato, I. (2000). Introducción a la Teoría General de la Administración. Colombia: McGraw Hill.

Delgado, A. \& Aliaga. (2007). Calidad de atención de enfermería desde la percepción del usuario que acude al servicio de emergencia del Hospital Nacional Guillermo Almenara Irigoyen. Revista de Ciencias de la Salud, 3(3), 67-68. Recuperado de: http://posgrado. upeu.edu.pe/revista/filev3/66-68.pdf

Dios, C., Alba, M., Ruíz, R., Jiménez, C., Pérula, L. \& Rodríguez, M. (2013). Valoración de la satisfacción de usuarios de consulta de Enfermería en Centros de Salud a partir de indicadores de calidad técnicos y de comunicación. Enferm. glob. 12 (31), 1-5.

Fernández, B., \& Paravic, T. (2003). Nivel de satisfacción laboral en Enfermeras de hospitales públicos y privados de la provincia de Concepción, Chile. Ciencia y enfermería. 9(2), 57-66. Recuperado en 17 de julio de 2014, de http://www.scielo.cl/scielo.php?script=sci arttext\&pid=S0717-95532003000200006\&lng=es\&tln $\mathrm{g}=$ es. 10.4067/S0717-95532003000200006.

García, C. Cortés, I. (2012). Percepción del usuario del trato digno por enfermería en un hospital del estado de México. Revista CONAMED. 17 (1), 18-23 Recuperado de: http:// dialnet.unirioja.es/servlet/articulo? codigo $=3884680$

Gómez, E. (2008). El cuidado de enfermería del paciente en estado crítico. Revista Científica Multidisciplinaria de Prospectiva. 21(2),1-3. Recuperado de: http://www. redalyc.uaemex.mx/pdf/832/83211487006.pdf

Herrera, G., \& Manrique, A., Fred, G. (2008). Condiciones laborales y grado de satisfacción de profesionales de enfermería. Aquichán, 8(2), 243256. Retrieved July 17, 2014, from http://www.scielo. org.co/scielo.php?script $=$ sci_arttext\&pid $=\mathrm{S} 1657$ 59972008000200011\&lng=en\&tlng=es.

Hernández, M., Ochando, A., Mora, J., Lorenzo, S., \& López, K. (2005). Satisfacción del paciente en una unidad de hemodiálisis. Rev. Soc. Esp. Enferm. Nefrol. 8(2): 90-96. Recuperadode:http://scielo.isciii.es/scielo.php?script=sci arttext\&pid=S113913752005000200002\&lng=es.

Iriarte (2012). Factores que influyen en la satisfacción laboral de enfermería en el concejo hospitalario de Navarra. Revista Electrónica de Enfermería. 1(17), 77-87 Recuperado de: http:/www.ucol.mx/egeneros/ admin/archivos/enfermeria_global.pdf
Kalisch, B., Lee, H. \& Rochman, M. (2010). Nursing staff teamwork and Job satisfaction. Journal of Nursing Management. 18(8), 938-947. DOI: 10.1111/j.13652834.2010.01153.x. Publicado:http://www.ncbi.nlm.nih. gov/entrez/eutils/elink.fcgi?dbfrom=pubmed\&retmode $=$ ref\&cmd $=$ prlinks\&id $=21073567$

Mahomed, R., St John, W., \& Patterson, E. (2012). Understanding the process of patient satisfaction with nurse-led chronic disease management in general practice. Journal of Advanced Nursing. 68(11): 2538-2549. DOI: 10.1111/j.1365-2648.2012.05953.x. Recuperado de: http://www.ncbi.nlm.nih.gov/ pubmed/22384855

Molina, E. (2011). Evaluación de la calidad del cuidado de enfermería en un servicio de hospitalización domiciliaria, Bogotá D.C. (Tesis Maestría). Universidad Nacional de Colombia. Bogotá. Recuperado de: http://www.bdigital. unal.edu.co/6471/1/Edithmilenamolinacardona.2011.pdf

Ortega, R. (2004). Percepción de la calidad del cuidado de enfermería según la opinión del paciente y la enfermera. (Tesis Maestría). Universidad Autónoma de Nuevo León. México. Recuperado de: http://cdigital. dgb.uanl.mx/te/1020149991.pdf

Parra, V., Guaqueta, P., \& Triana, P. (2012) Perspectiva del cuidado de enfermería en UCI una visión desde el modelo de cuidado HANC. Colombia: Editorial Kimpres.

Ponce, J., Reyes, M. y Ponce, G. (2006). Satisfacción laboral y calidad de atención de enfermería en una unidad médica de alta especialidad. Rev. Enferm. 14(2), 5-63. Recuperado de http://www.medigraphic.com/ pdfs/enfermeriaimss/eim-2006/eim062b.pdf

Ruzafa, M., Madrigal, M., Valandrino, A. \& López, L. (2008). Satisfacción laboral de los profesionales de enfermería españoles que trabajan en los hospitales ingleses. Gaseta sanitaria. 22(5), 3-5. Recuperado el 17 de julio, de: http://scielo.isciii.es/scielo.php?script=sci arttext\&pid $=$ S0213-91112008000500007

Sarella, L. H., \& Paravick, T. (2002). Satisfacción laboral en enfermeras/os que trabajan en el sistema de atención médica de urgencia (SAMU). Ciencia y enfermería, 8(2), 37-48. Recuperado en 16 de julio de 2014, de http://www.scielo.cl/scielo.php?script=sci arttext\&pid=S0717-95532002000200005\&lng=es\&tln $\mathrm{g}=$ es. $10.4067 / \mathrm{S} 0717-95532002000200005$

Serrano-del Rosal, R., \& Loriente-Arín, N. (2008). La anatomía de la satisfacción del paciente. Salud Pública de México, 50(2), 162-172. Recuperado en 16 de julio de 2014, de http://www.scielo.org. $\mathrm{mx} / \mathrm{scielo} . \mathrm{php}$ ? script $=\mathrm{sci}$ arttext\&pid $=\mathrm{S} 0036$ 36342008000200010\&lng=es\&tlng=es. 